\title{
El aprendizaje orientado en proyectos para el desarrollo de habilidades blandas en el nivel medio superior del IPN
}

\author{
Project-Based Learning for the Development of Soft Skills in the Upper \\ Secondary Level of the Instituto Politécnico Nacional
}

\section{Aprendizagem orientada a projetos para o desenvolvimento de habilidades} sociais no nível médio superior do IPN

María Elena Zepeda Hurtado

Instituto Politécnico Nacional, México

mezepedah@ipn.mx https://orcid.org/0000-0001-9764-5013

Edgar Oliver Cardoso Espinosa Instituto Politécnico Nacional, México eoce@hotmail.com https://orcid.org/0000-0001-7588-9439

Jésica Alhelí Cortés Ruiz

Instituto Politécnico Nacional, México jacr2709@hotmail.com https://orcid.org/0000-0002-5459-4874

\section{Resumen}

El objetivo de esta investigación fue desarrollar en estudiantes de nivel medio superior del Instituto Politécnico Nacional (IPN) habilidades blandas mediante la implementación de la metodología del aprendizaje orientado en proyectos. El tipo de estudio que se empleó fue una metodología cuantitativa con un alcance descriptivo. El instrumento que se utilizó para obtener la información fue un cuestionario basado en una escala Likert. La muestra del estudio estuvo constituida por el alumnado inscrito en el Centro de Estudios Científicos y Tecnológicos perteneciente al IPN. La distribución de la muestra con respecto al género fue de $67 \%$ de hombres y $33 \%$ mujeres. Los principales resultados fueron que los participantes reconocieron 


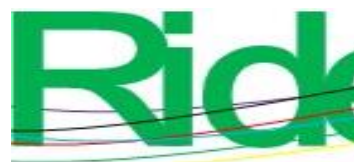

Revista Iberoamericana para la

Investigación y el Desarrollo Educativo ISSN $2007-7467$

un desarrollo muy frecuente de sus habilidades blandas en cuatro rubros: interpersonales, toma de decisiones, pensamiento crítico y autocontrol, y habilidades comunicativas.

Palabras clave: aprendizaje basado en proyectos, educación media superior, estudiantado, habilidades blandas.

\section{Abstract}

The objective of this research was to develop soft skills in students through the implementation of the project-based learning methodology at the upper secondary level of the Instituto Politécnico Nacional (IPN). It was used a quantitative methodology with a descriptive scope. The instrument that was used to obtain the information was a questionnaire based on a Likert scale. The sample of the study was constituted by the students enrolled in the Centro de Estudios Científicos y Tecnológicos belonging to the IPN. The sample distribution with respect to gender was $67 \%$ of men and the remaining $33 \%$ of women. The main results were that the participants recognized a very frequent development of their soft skills in the following categories: interpersonal, decision making, critical thinking and self-control, and communication skills.

Keywords: project-based learning, upper secondary education, student body, soft skills.

\section{Resumo}

O objetivo desta pesquisa foi desenvolver habilidades sociais em estudantes do nível médio superior do Instituto Politécnico Nacional (IPN), implementando a metodologia de aprendizagem orientada a projetos. O tipo de estudo utilizado foi uma metodologia quantitativa, com escopo descritivo. O instrumento utilizado para obter as informações foi um questionário baseado na escala Likert. A amostra do estudo foi composta pelos alunos matriculados no Centro de Estudos Científicos e Tecnológicos pertencentes ao IPN. A distribuição da amostra em relação ao gênero foi de $67 \%$ homens e $33 \%$ mulheres. Os principais resultados foram que os participantes reconheceram um desenvolvimento muito frequente de suas habilidades sociais em quatro áreas: interpessoal, tomada de decisão, pensamento crítico e autocontrole e habilidades de comunicação.

Palavras-chave: aprendizagem baseada em projetos, ensino médio, estudantes, habilidades sociais.

Fecha Recepción: Mayo 2019

Fecha Aceptación: Septiembre 2019 


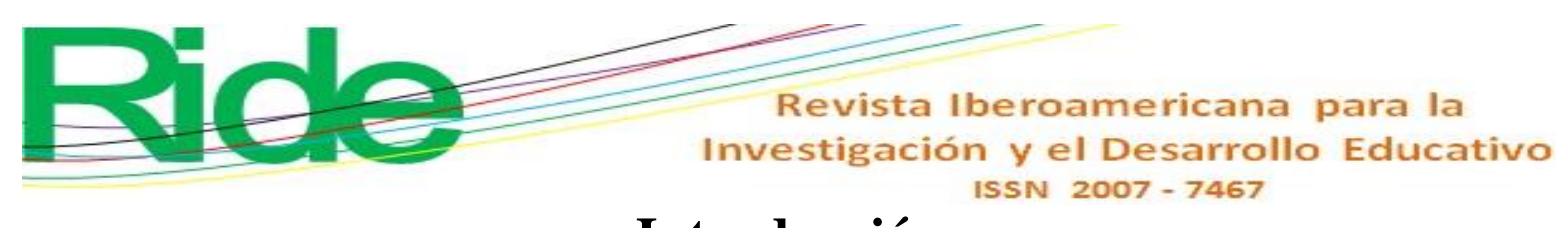

\section{Introducción}

Teniendo en cuenta que la educación posee un papel fundamental en el desarrollo de una sociedad, los sistemas educativos requieren considerar sus necesidades y demandas, así como los intereses de los ciud

adanos y las metas que como país se pretenden alcanzar. Actualmente los docentes se enfrentan al desafío de formar personas que se van a desempeñar en una sociedad caracterizada por la incertidumbre, la innovación y la circulación de información a partir del empleo de las herramientas tecnológicas, por lo que es preciso que las nuevas generaciones aprendan a vivir y desenvolverse en este contexto (Bauman, 2008).

En ese sentido, la Organización para la Cooperación y el Desarrollo Económicos [OECD, por sus siglas en inglés] (2017) estableció lo puesto a continuación:

En el siglo XXI, las competencias, destrezas y habilidades se han convertido en el factor clave para lograr el bienestar individual y el éxito económico de una sociedad. Sin una inversión adecuada en estos ámbitos, la población languidece al margen de la sociedad, el progreso tecnológico no se transforma en crecimiento y los países son incapaces de competir en una economía mundial que se basa cada vez más en el conocimiento (p. 1).

De este modo, la educación ha respondido a los cambiantes requerimientos de la sociedad y de la industria. Esto representa tanto para las instituciones como para el profesorado el compromiso de estar al día para enriquecer la formación integral de los alumnos y formar egresados que, además de tener una formación disciplinaria, sean creativos, innovadores y proactivos. Bajo esta perspectiva, es necesario comprender, desarrollar y poner en práctica estrategias didácticas en el aula con la intención de realizar experiencias de aprendizaje que fortalezcan y desarrollen habilidades, destrezas y actitudes para la investigación, el trabajo colaborativo, el empleo de las tecnologías de la información y la comunicación (TIC), que permitan la generación de propuestas de solución a problemas tanto de la vida personal como laboral.

Por lo que no es de extrañar la influencia cada vez mayor de las TIC en todos los niveles del sistema educativo. Al respecto, Marcovitch (2002) menciona: “Ante la revolución tecnológica, las instituciones de educación superior (IES) se comportan como cualquier otra organización que no puede ignorar y dejar de aprovechar sus beneficios” (p. 87).

En el World Economic Forum [WEF] (2017) se identificaron diversos cambios tecnológicos disruptivos que están transformando las relaciones sociales entre humanos y objetos. Así, se ha dado el uso generalizado de Internet, el empleo absoluto de los dispositivos móviles tanto a nivel personal como organizacional, el censo poblacional mediante el big data, 


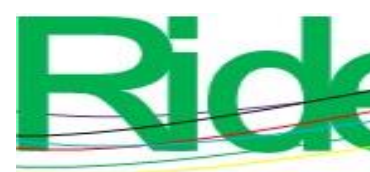

Revista Iberoamericana para la

Investigación y el Desarrollo Educativo ISSN $2007-7467$

la recaudación de impuestos por medio de blockchain, así como incorporar la inteligencia artificial en las reuniones directivas. A este conjunto de cambios se le ha nombrado la cuarta revolución industrial. Dicha revolución se caracteriza por el empleo de las nuevas tecnologías exponenciales como la robótica avanzada, el transporte autónomo, la inteligencia artificial, los sensores de recogida de datos, la Internet de los objetos, la manufactura por impresión 3D, la nanotecnología o la computación cuántica (Coleman, 23 de enero de 2016).

De acuerdo con Escudero (2018), la primera revolución industrial surgió a finales del siglo XVIII en el Reino Unido, con la irrupción de la energía de vapor, la mecanización de la agricultura, la manufactura y el transporte (Daemmrich, 2017). La segunda revolución industrial se consolidó en Estados Unidos de América a principios del siglo XX, en donde la tecnología disruptiva fue la energía eléctrica, que permitió la producción en serie (Hintz, 2011; MacLeod, 2016). La tercera revolución industrial fue detonada desde mediados del siglo pasado con la invención de los semiconductores, las plataformas digitales y el auge de las computadoras personales (Hermann, Pentek y Otto, 2016). A diferencia de las tres revoluciones anteriores, la cuarta no se define por la emergencia de una tecnología disruptiva específica, sino por la convergencia de varias tecnologías digitales, físicas y biológicas, como la inteligencia artificial, la inteligencia aumentada, la robótica, la impresión 3D, el cloud computing, el big data, el Internet de las cosas o la nanotecnología (Rose, 2016).

De esta forma, las revoluciones industriales implantaron equipos, herramientas, materiales y recursos de diversas índoles, desde las máquinas de vapor y la energía eléctrica hasta llegar a la electrónica y la informática, lo que ha ocasionado en las industrias, primero, producciones mecanizadas y, más tarde, automatizadas. Y evidentemente el impacto no solo fue en el ámbito laboral o industrial sino en la vida cotidiana.

Particularmente, lo que caracteriza a la cuarta revolución industrial son las formas de producción basadas en el uso de sistemas físicos cibernéticos para crear una industria más flexible y de carácter reconfigurable, es decir, que la estructura de una fábrica se pueda modificar para poder producir diferentes productos a partir del empleo del big data y análisis de datos, los robots autónomos, la simulación de procesos en computadora, sistemas de integración, Internet de las cosas aplicado a la industria, ciberseguridad, almacenamiento de información en la nube, impresión 3D o manufactura aditiva y la realidad aumentada (Capilla, 3 de octubre de 2017). Asimismo, Joyanes (2018) y el WEF (2017) identificaron la incorporación en la sociedad del uso de diversas tecnologías emergentes como son los nanomateriales, la robótica, las biotecnologías, las redes eléctricas inteligentes, los sistemas criptográficos, la ciberseguridad, entre otras ya mencionadas anteriormente. 


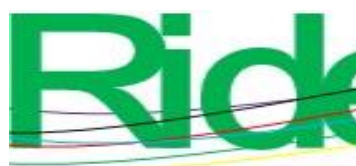

Revista Iberoamericana para la

Investigación y el Desarrollo Educativo ISSN $2007-7467$

Lo anterior implica una alta inversión en tecnología, y por extensión también implica el desarrollo de competencias del personal, ya que los trabajadores deberán adquirir un nuevo conjunto de capacidades relacionadas con el manejo y análisis de datos, la producción asistida por computadora, simulación en línea, programación, mantenimiento predictivo y similares (Ynzunza, 2017).

Cabe mencionar que tener conocimiento sobre los avances tecnológicos en la industria trae como consecuencia una mayor claridad sobre las implicaciones, beneficios y retos en otros ámbitos que marcarán la vida cotidiana: formas de vivir, comunicarse, empleos, negocios. En el reporte del WEF (2016), cuyo título traducido al español es El futuro de los trabajos. Empleos, habilidades y estrategias de fuerza laboral para la cuarta revolución industrial, entre otras aportaciones se manifiesta que los modelos de negocios tendrán un alto impacto en los empleos; desafío que requiere de adaptación proactiva por parte de las industrias, gobiernos e instituciones, debido a que los empleos sufrirán una transformación fundamental en categorías y ocupaciones, cambiarán las habilidades requeridas por estos: se demandarán habilidades que mejoren la productividad y los libere del trabajo rutinario, lo que trae como consecuencia reinventar las funciones de los recursos humanos: desarrollo de habilidades, gestión de talento, desempeño de roles multifuncionales. Mientras que para algunos es preocupante por la pérdida de empleos, para otros es un área de oportunidad, un terreno fértil de nuevos empleos.

Ante este escenario, es necesaria una transformación de los sistemas educativos: se requiere un ambiente de aprendizaje que promueva el desarrollo de capacidades como fundamento de la formación, cuyo enfoque permita al individuo resolver una situación compleja a partir de estrategias de solución que generen habilidades, destrezas y actitudes (Pavié, 2011).

\section{Fundamentación teórica}

La OECD (2012) define a las competencias como el conjunto de conocimientos, habilidades y destrezas que pueden aprenderse; conjunto que permite a los individuos realizar una actividad o tarea de manera adecuada y sistemática, y que puede adquirirse y ampliarse a través del aprendizaje. Esta definición incluye toda la gama de competencias cognitivas, tal y como la alfabetización y la aritmética; técnicas que son específicas de un sector u ocupación, y las socioemocionales, relacionadas con el trabajo en equipo y la comunicación. Por lo que el conjunto de todas las competencias disponibles para la economía en un momento dado conforma el capital humano de un país.

En tanto la Organización de las Naciones Unidas para la Educación, la Ciencia y la Cultura [Unesco] (2015) ha propuesto establecer programas de vinculación escuela-industria o escuela-empresa con el objetivo de desarrollar competencias flexibles necesarias a la realidad 


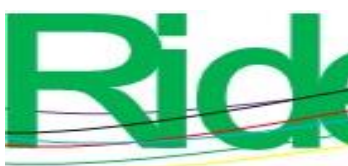

Revista Iberoamericana para la

Investigación y el Desarrollo Educativo ISSN $2007-7467$

cambiante del mundo laboral, y que también se conocen como transferibles o no cognoscitivas.

Dichas capacidades son la comunicación, la alfabetización digital, la resolución de problemas, el trabajo en equipo y el espíritu de empresa.

Por tanto, en el corto plazo, se necesitarán graduados en diferentes áreas, principalmente digitales. De acuerdo con la Secretaría de Estrategias Industriales de Madrid (2017), también se deben incluir competencias transversales, como el trabajo en equipo, la capacidad de adaptación y emprendimiento, o la buena expresión oral y en público. No hay que olvidar que el sistema educativo no tiene como única finalidad alimentar al mercado laboral con lo que demande en cada momento, sino que tiene que producir ciudadanos críticos, cultos e inteligentes, con formaciones integrales en sus especialidades.

Aunado a lo ya dicho, la OECD (2017) ha establecido que las principales competencias para el desempeño laboral forman un continuo, desde habilidades que son en su mayoría cognitivas a habilidades en su mayoría vinculadas a rasgos de personalidad, con actitudes como se muestra en la figura 1.

Figura 1. Competencias relevantes

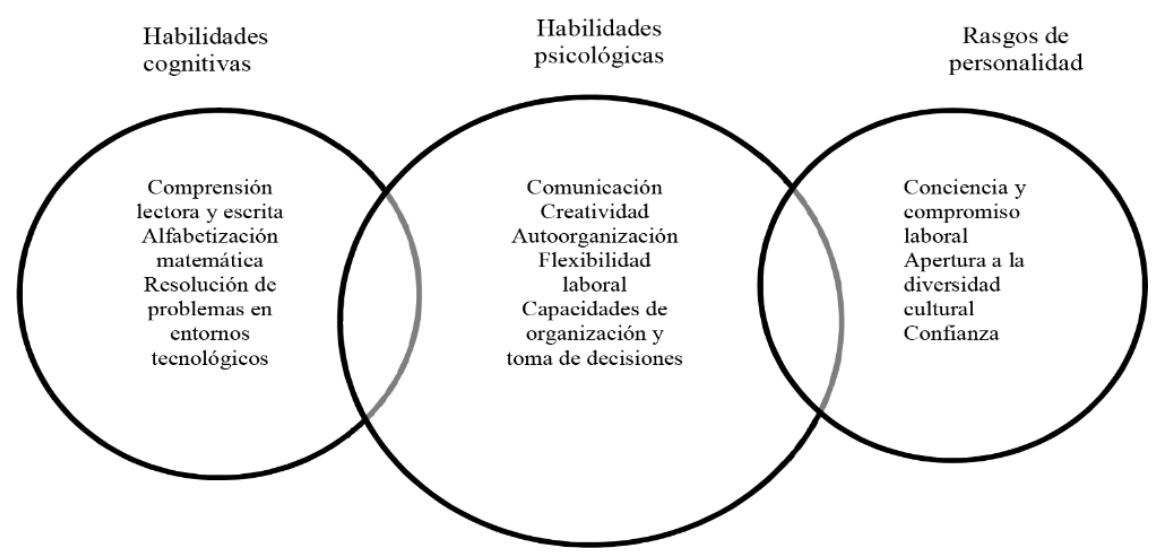

Fuente: OECD (2017)

Como lo señala el WEF (2018): las competencias relevantes a desarrollar en las personas para el siglo XXI desde la educación básica hasta la superior son la resolución de problemas complejos, el pensamiento crítico, la creatividad, la colaboración y la alfabetización digital, las cuales son fundamentales para permitir a las personas seguir siendo flexibles y adaptables a las necesidades cambiantes de la sociedad. Específicamente, en este foro se hicieron recomendaciones referidas al desarrollo de las habilidades laborales para la cuarta revolución industrial que serán trascendentales para el futuro inmediato, de acuerdo con su proyección para el 2020. Se trata de las siguientes: 1) Creatividad; 2) Gestión de personas; 3) Coordinación con los demás; 4) Inteligencia emocional; 5) Juicio y toma de decisiones; 6) Orientación de servicio; 7) Negociación y 8) Flexibilidad cognitiva (WEF, 2018). 


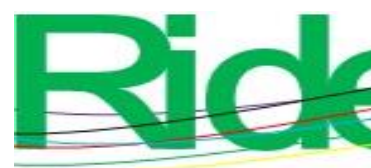

Revista Iberoamericana para la

Investigación y el Desarrollo Educativo ISSN $2007-7467$

También la Unesco (2018) estableció la necesidad de integrar la modalidad educativa llamada dual (que combina la enseñanza en el espacio laboral en una forma estructural) y cuyo enfoque es integral debido a que no solo genera conocimientos técnicos, sino también las “competencias blandas" (inteligencia emocional, autocontrol, trabajo en equipo, perseverancia y liderazgo) para asegurar que aquellos jóvenes que consiguen un empleo logren conservarlo.

Por consiguiente, las competencias blandas, también conocidas como soft skills, son consideradas como un componente relevante en una formación tanto transversal como laboral debido al alto nivel de interrelación que se establece mediante las redes sociales y otras tecnologías, que convierten al entorno en un constante intercambio de datos, información, comunicación y conocimiento (Ortega, 2016). De esta forma, los profesionales que posean una alta integración de habilidades blancas y competencias técnicas tendrán menor riesgo de ser sustituidos por procesos automáticos que tienen limitaciones de interacción (David, 2015; Frey y Osborne, 2017).

Por su parte, Murti (2014) indica que las habilidades blandas se han determinado como necesarias en el ambiente laboral debido a que son transversales, porque están relacionadas con la personalidad, actitud y comportamiento de cada persona. Así, el perfil de un egresado tiene que estar preparado para el aprendizaje permanente y también debe poseer una buena comunicación y una alta capacidad de trabajo en equipo, ya que, una vez más, las competencias técnicas no son suficiente (Mafflioli y Giuliano, 2003).

Del mismo modo, en el estudio realizado por Dahm, Farrell y Ramachandran (2015) se determinó que aquellos profesionales que tienen mayores habilidades de comunicación son percibidos con mayor capacidad técnica, lo cual se manifiesta en la facilidad de encontrar empleo. En tanto que en la investigación efectuada por Deming (2015) se encontró que la demanda en el mercado laboral global de habilidades blandas ha tenido un crecimiento de 24 $\%$ porque, siguiendo esta misma fuente, se ha reconocido como imprescindible que las habilidades técnicas se integren con las blandas, con un enfoque de trabajo multidisciplinario. Asimismo, Robles (2012) estableció que las habilidades de comunicación oral y escrita, aunado al trabajo en equipo, tienen una alta correlación con el éxito profesional.

Para el caso de México, los niveles de la educación media superior son relativamente bajos. El índice de escolarización de estudiantes de 15 a 19 años disminuye a $54 \%$, que lo ubica en la última posición de los países de la OECD y debajo de la media de la organización, que es de $84 \%$. Asimismo, solamente $16 \%$ de los adultos de 25 a 64 años ha cursado estudios de tipo superior: la proporción más baja de los países de la OECD. Por tanto, México tiende a especializarse en actividades de bajo valor agregado, lo que genera empleo informal, de baja calidad y remuneración. Para contrarrestar esta situación, es necesario brindar oportunidades 


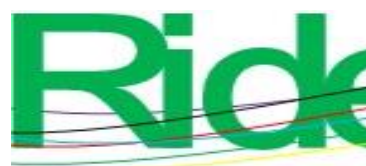

Revista Iberoamericana para la

Investigación y el Desarrollo Educativo ISSN 2007 - 7467

para que los estudiantes de los niveles de media superior desarrollen competencias relevantes.

Esto permitirá sentar las bases para construir una sociedad más equitativa e incrementará los niveles de productividad del país.

En este sentido, es necesario desarrollar competencias básicas sólidas que le permitan a los individuos insertarse al mercado laboral o transitar a la educación superior: y para lograrlo el nivel antecedente se constituye indudablemente en una piedra angular. Así, los egresados contarán con mejores competencias y una mayor productividad, lo que incrementa las posibilidades de encontrar trabajo en la economía formal y de obtener salarios más altos. Una población con mayores niveles de educación fomenta la prosperidad económica y mejores niveles de vida (OECD, 2017).

Para lograr el desarrollo de las habilidades blandas, autores como Dole, Bloom y Kowalske (2016), Freeman et al. (2014), al igual que Al-Balushi y Al-Amri (2014), establecieron la necesidad de emplear un enfoque didáctico innovador en el desarrollo del proceso de enseñanza-aprendizaje orientado a que el estudiantado investigue desde sus conocimientos previos en un ambiente colaborativo. Además, en el proceso de formación de competencias son importantes los contenidos a desarrollar en el alumnado, pero lo es también imprescindible la actuación metodológica que se lleva a cabo, es decir, la forma de abordarlos por parte del profesorado (Blanco, Jové y Reverter, 2012).

Así, se han empezado a emplear metodologías didácticas activas con la finalidad de conseguir una mayor participación del alumnado en su aprendizaje, las cuales se orientan a brindar un mayor protagonismo por parte del estudiante a partir de hacer, indagar e interactuar utilizando las TIC (Konopka, Adaime y Mosele, 2015). En ese sentido, las metodologías activas son el conjunto de actividades y estrategias que usa el profesorado para conseguir una mayor participación del alumno en la construcción de su aprendizaje. Y entre este tipo de acercamientos pedagógicos destaca el aprendizaje orientado en proyectos (AOP), el aprendizaje basado en problemas (ABP), la gamificación y el aula invertida (Roig y Álvarez, 2019).

Al respecto del AOP, del cual nos centraremos en este artículo, ofrece una alternativa que favorece el tratamiento de los contenidos con el entorno e intereses del alumno (Merrit, Lee, Rillero y Kinach, 2017; Rosa y Martínez, 2016). Markham, Larner y Ravitz (2003) y Blanchard y Dolores (2016) definen que el AOP es un método sistemático de enseñanza que involucra a los estudiantes en la adquisición de conocimientos y habilidades a través de un proceso extendido de indagación, estructurado alrededor de preguntas para la elaboración de un producto determinado, por lo que, al articular la teoría y la práctica, genera conocimientos significativos. Por su parte, Vergara (2015) lo conceptualiza como un proceso de aprendizaje 


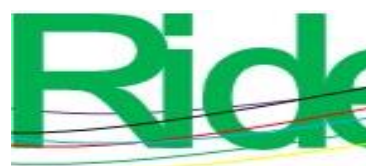

Revista Iberoamericana para la

Investigación y el Desarrollo Educativo ISSN $2007-7467$

basado en la investigación, los principios de negociación igualitaria, la responsabilidad individual, la actitud de cooperación y la toma de decisiones consensuada.

En este sentido, el AOP es considerada como una de las principales estrategias didácticas que permite el desarrollo de diversas competencias, tales como la creatividad, resolución de problemas, habilidad para investigar, trabajar de forma colaborativa, motivación y uso de redes sociales que se van a potenciar en los sistemas educativos del siglo XXI (Austin, Abella, Delgado y Hortigüela, 2016; Landron, Agreda y Colmenero, 2018). Consecuentemente, para la implementación del AOP en la actualidad es imprescindible una organización de los espacios, los materiales y los recursos, así como la inclusión de las TIC para un impacto efectivo en el desarrollo de las competencias del estudiantado (Cascales y Carrillo, 2018).

Otros investigadores que se han ocupado de esta metodología han sido Willard y Duffrin (2003) y Carrasco, Donoso, Duarte, Hernández y López (2015), quienes establecieron que es eficaz para el desarrollo de habilidades en la solución de situaciones de la vida real que implican el empleo también de actitudes y valores que favorecen la capacidad de aprendizaje a lo largo de la vida. Así, esta metodología permite fomentar la adquisición de conocimientos, la comunicación oral y escrita, el pensamiento crítico, el trabajo en equipo, la planeación y gestión del tiempo, aunado a la responsabilidad individual y grupal (De Miguel, 2006; Álvarez, Herrejón, Morelos y Rubio, 2010).

Según Thomas (2010) y Vergara (2015), las características del AOP son las siguientes: parte de situaciones relacionadas con la futura profesión de los estudiantes; la planeación de actividades para elaborar un producto; responde a los intereses y necesidades de los alumnos; el resultado o producto es valioso y útil; los estudiantes desarrollan con autonomía acciones específicas articulando la teoría y práctica; las actividades de las diferentes fases del proyecto son decidas y ejecutadas por los propios alumnos, y se fomenta el trabajo en equipo, por lo que el aprendizaje es holístico e integral. En tanto que las guías del AOP son las siguientes: la enseñanza se produce en el contexto de una situación; los contenidos clave se aprenden a partir del contexto y la actuación, aunado a que se inicia con un propósito compartido por el alumnado (Doménech-Casal, 2018).

Para llevar a cabo el AOP es necesario que los estudiantes trabajen de forma autónoma en la realización de un proyecto real durante un periodo de tiempo determinado, en donde se requiere la planeación, el diseño y la realización de una serie de actividades en las que es necesario aplicar diversas habilidades, actitudes, así como usar de forma efectiva los recursos al alcance mediante la reflexión, el debate, comunicación para recolectar y analizar la información y la generación de sus propias estrategias de resolución (Mettas y Constantinou, 2007). De este modo, un proyecto aborda la realidad para que los alumnos la analicen, la 


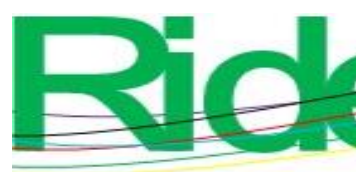

Revista Iberoamericana para la

Investigación y el Desarrollo Educativo ISSN $2007-7467$

empleen como herramienta de aprendizaje, intervengan en ella, por lo que su relevancia genera experiencias educativas que provocan un cambio personal y grupal (Vergara, 2016).

Por tanto, los beneficios que tiene son los siguiente: vincula el saber conocer con el saber hacer; posibilita el fomento de las habilidades de solución de situaciones, comunicación y autonomía; incentiva el aprendizaje a lo largo de la vida, la responsabilidad social y el éxito personal; genera una comunicación proactiva y relaciones de colaboración; considera las necesidades de los alumnos con sus estilos, y motiva a los alumnos apáticos (Markham et al. 2003). En este sentido, el AOP permite establecer las condiciones que conducen a un aprendizaje activo, contextualizado, integrado y orientado a la comprensión, lo que brinda oportunidades para reflexionar sobre la experiencia y aplicar lo aprendido a partir de una situación a enfrentar (Alonso, 2018). Como se puede ver, el AOP enfatiza el protagonismo del estudiante en su proceso de aprendizaje, la comunicación interpersonal, la inventiva, la formulación y resolución de situaciones, la búsqueda de información continua con la finalidad de comprender su realidad (Rodríguez, Vitvitskaya y Silva, 2018). A razón de lo anterior ha sido investigado como una propuesta que posibilita la mejora del aprendizaje convencional.

En suma, la finalidad del AOP es generar una mayor motivación tanto en los temas disciplinares como en el desarrollo de las habilidades, debido a que la generación de un proyecto exitoso requiere que el alumnado utilice sus competencias de comunicación, trabajo en equipo, gestión del tiempo y liderazgo (Davcev, Stojkoska, Kalajdziski y Trivodaliev, 2016).

De este modo, integra con mayor impacto los diferentes temas teóricos y prácticos, por lo que el estudiante implementa lo aprendido en la creación y desarrollo de un proyecto, gracias a lo cual, a su vez, se promueve la motivación intrínseca y se incrementa la creatividad (Hernando, Galán, Navarro y Rodríguez, 2011; Neebel, Merkel y Wong, 2013). Asimismo, el AOP genera en el alumnado la búsqueda, análisis e integración de la información con la finalidad de elaborar una evidencia apropiada con el proyecto por lo que la competencia oral y escrita se convierte en un proceso de apropiación y manejo trascendental como una práctica discursiva en la que se difunde y manifiesta el modo de pensamiento de un ámbito disciplinar determinado (Álvarez y Boillos, 2015).

No obstante, se requieren estudios específicos sobre las diversas asignaturas y niveles educativos para determinar los efectos del AOP sobre el desempeño académico del alumnado (Landron et al., 2018). 


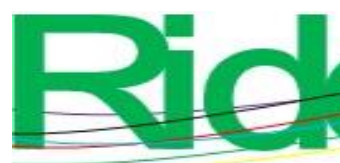

Revista Iberoamericana para la Investigación y el Desarrollo Educativo ISSN $2007-7467$

lo inicialmente esperado: el uso del proyecto asociado a una problemática les generó un mayor interés y responsabilidad.

La investigación llevada a cabo por Alvarado, Galindo y Retamal (2018) destaca por el hecho de que $69.6 \%$ de los estudiantes participantes contextualizó y fundamentó su proyecto con documentos disponibles en Internet; el total de los participantes elaboró el informe del proyecto; $96 \%$ desarrolló la comunicación oral y escrita, y $85 \%$ valoró positivamente el empleo de la AOP en su formación académica debido a que permitió integrar el conocimiento, el uso de recursos informáticos, el pensamiento crítico.

Finalmente, Rico, Garay y Ruiz (2018) concluyeron que la implementación del AOP influyó de forma positiva en la formación del alumnado en lo que respecta a su nivel de conocimientos y habilidades de investigación, aunado al desarrollo de capacidades de análisis y síntesis. También generó un trabajo en equipo que permitió compartir los retos y logros con sus compañeros, así como la inserción de las TIC para resolver la situación y se reconoció su relevancia en el aprendizaje.

\section{Objetivo del estudio}

Desarrollar en estudiantes de nivel medio superior del Instituto Politécnico Nacional (IPN) habilidades blandas mediante la implementación de la metodología del AOP.

\section{Método}

\section{Tipo de estudio}

El tipo de estudio que se utilizó fue una metodología cuantitativa con un alcance descriptivo porque se orientó determinar los niveles de desarrollo de las habilidades blandas en el alumnado de educación media superior del IPN (Namakforoosh, 2011).

Para desarrollar las habilidades blandas se implementó la estrategia del AOP en la unidad de aprendizaje de Expresión oral y escrita I, que pertenece al primer semestre de bachillerato. Dichas habilidades se agruparon en una serie de categorías (ver tabla 1). 


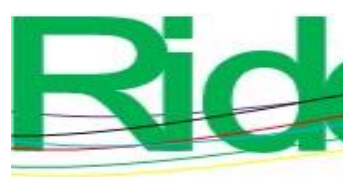

Revista lberoamericana para la

Investigación y el Desarrollo Educativo ISSN $2007-7467$

Tabla 1. Habilidades blandas del estudio

\begin{tabular}{|l|l|l|l|}
\hline $\begin{array}{l}\text { Habilidades } \\
\text { interpersonales }\end{array}$ & $\begin{array}{l}\text { Habilidades de } \\
\text { toma de decisión } \\
\text { y pensamiento } \\
\text { crítico }\end{array}$ & $\begin{array}{c}\text { Habilidades de } \\
\text { autocontrol y } \\
\text { afrontamiento }\end{array}$ & $\begin{array}{c}\text { Habilidades } \\
\text { comunicativas }\end{array}$ \\
\hline $\begin{array}{l}\text { Comunicación } \\
\text { interpersonal }\end{array}$ & $\begin{array}{l}\text { Resolución de } \\
\text { problemas }\end{array}$ & $\begin{array}{l}\text { Aumentar la } \\
\text { confianza personal }\end{array}$ & Escuchar \\
\hline $\begin{array}{l}\text { Negociación } \\
\text { equipo }\end{array}$ & $\begin{array}{l}\text { Toma de } \\
\text { decisiones }\end{array}$ & Responsabilidad & Leer \\
\hline $\begin{array}{l}\text { Desarrollo de la } \\
\text { creatividad }\end{array}$ & $\begin{array}{l}\text { Liderazgo } \\
\text { metigación }\end{array}$ & Expresión oral \\
\hline
\end{tabular}

Fuente: Leyva (2018)

\section{Descripción de la situación educativa basada en el AOP}

La situación educativa se implementó con el alumnado de primer semestre de bachillerato, enfocándose en el desarrollo de una investigación de un fenómeno real como es la migración, y que culminó con la presentación de los resultados. La unidad de aprendizaje seleccionada fue la de Expresión oral y escrita I, cuya competencia general a lograr es que el alumno utilice mensajes informativos, de manera oral y escrita, empleando correctamente la gramática española en diversas situaciones comunicativas.

Para alcanzar dicha competencia se establecen dentro del programa de estudios tres unidades con su respectiva competencia particular, cada una integrada por dos resultados de aprendizaje propuestos (RAP) que se enuncian a continuación:

- $\quad$ Expresa ideas y/o emociones en diversas situaciones comunicativas respetando las formas básicas de la lengua escrita.

RAP 1: Muestra las características, semejanzas y diferencias entre el lenguaje, la lengua y el habla, ejemplificándolas en diversos textos informativos.

RAP 2: Analiza diversos mensajes describiendo el nivel de la lengua a los que pertenecen.

- Redacta textos informativos haciendo uso de los modelos expositivos para expresar ideas y/o emociones.

RAP 1: Describe las cualidades de la expresión escrita y las características de los modelos expositivos ejemplificándolos en diferentes textos. 


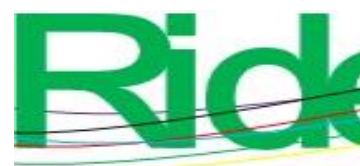

Revista Iberoamericana para la

Investigación y el Desarrollo Educativo ISSN $2007-7467$

RAP 2: Construye textos expositivos empleando las características propias de los modelos.

- $\quad$ Expone temas o asuntos empleando las cualidades de la expresión oral para manifestar ideas y/o emociones.

RAP 1: Identifica las cualidades y técnicas de expresión oral para emplearlas en diversas situaciones comunicativas.

RAP 2: Emplea la expresión oral en función de sus cualidades y de la variedad de las técnicas de exposición para comunicar ideas y/o sentimientos.

Específicamente, la metodología del AOP para este curso se implementó de la forma que se expone líneas más adelante, y bajo la consideración de que es un proceso en el que se usan métodos y técnicas de la investigación documental, por lo que, además, se incorporaron las competencias antes mencionadas, lo que permitió desarrollarlas para la elaboración de una propuesta de solución de al problema específico de la migración.

De esta manera, las fases de la metodología del AOP para este estudio con sus respectivas actividades fueron las siguientes:

1. ${ }^{a}$ fase: Asamblea con los integrantes del grupo para la elección y delimitación del tema, destacando propuestas enfocadas acerca de la prevención de adicciones; promoción al cuidado de la salud; equidad y género; derechos humanos; interculturalidad; violencia social; ciencia, tecnología y educación; desarrollo sustentable y emprendimiento.

En una segunda sesión, ya teniendo conocimientos previos de los ejes temáticos, se discutió y decidió por uno solo con la finalidad de llevar a cabo su delimitación y justificación, de lo cual surgió el planteamiento de una situación por resolver en un contexto específico, y que fue la interculturalidad, la cual se orientó en indagar qué sucede con el fenómeno de la migración. Debido a que actualmente en todo el mundo se han acentuado los flujos migratorios: personas que buscan mejores condiciones de vida y oportunidades de crecimiento más allá de sus lugares de origen, ya sea por cuestiones laborales, educativas, económicas, políticas y salariales. Sin embargo, en el trayecto de un lugar a otro son víctimas de extorsiones, violaciones, maltrato y desapariciones, independientemente de la edad o sexo.

2. ${ }^{a}$ fase: Búsqueda y selección de información relevante en torno al problema de la inmigración en artículos de revistas y libros científicos, así como realización de entrevistas a expertos.

En este sentido, para la investigación documental, se consideraron las características de factibilidad, novedad e importancia. Así, el estudiantado se fue orientando para elaborar el marco teórico y comprobar la hipótesis planteada, analizar e informar de los resultados. Teniendo información general del tema, el grupo fue subdividido en equipos, para que cada uno 


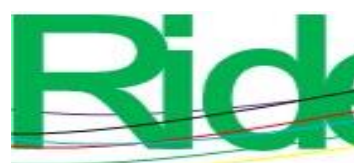

Revista Iberoamericana para la Investigación y el Desarrollo Educativo ISSN $2007-7467$

investigara las causas y consecuencias de la migración en los países que presentan mayor porcentaje de este fenómeno, como son Siria, India, México, Israel, Palestina Afganistán y Venezuela. Durante este proceso, se dio seguimiento a la entrega y revisión de los avances de la investigación por parte del docente.

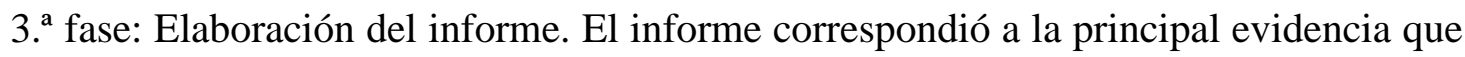
está íntimamente relacionada con el desarrollo de competencias de la segunda unidad de aprendizaje, y que hace referencia a la redacción de textos informativos basados en el uso de los modelos expositivos: cronológico, causa-efecto, tesis-antítesis y síntesis. Dicho documento permitió que los equipos elaboraran propuestas de solución con la finalidad evaluar la factibilidad de cada una de ellas.

Asimismo, en la formulación de la evidencia se cuidó el empleo de las cualidades de la redacción, así como que cumpliera con los componentes de la estructura mínima de un texto informativo: introducción, desarrollo y conclusiones.

4. ${ }^{a}$ fase: Selección de la mejor estrategia para generar la evidencia final: noticiero. Una vez realizado el informe, el último paso consistió en decidir la forma de presentar los resultados de manera creativa e innovadora. En plenaria, el grupo, después de una discusión fundamentada, decidió que cada equipo realizara un noticiero, por lo que fue necesario investigar, a través de la observación de diversos noticieros, tanto sus características como su estructura.

Además, para dar seguimiento a la planeación del noticiero, se solicitó un guion de equipo y uno individual; aquel reflejara la estructura del noticiero: el nombre, lema, secciones, cortinillas, presentadores, corresponsales o reporteros; y este último, la información que cada uno iba a presentar durante su participación.

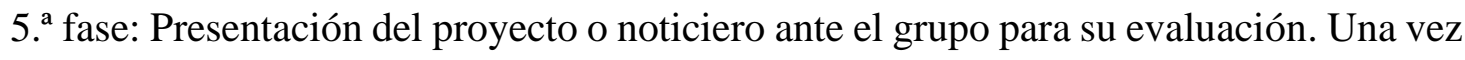
realizado el cronograma de presentaciones, cada equipo realizó la presentación para después hacer una coevaluación. Así, los resultados expresados por los alumnos fueron que el proyecto favoreció la formación académica y personal, identificando las habilidades y actitudes que se crearon o desarrollaron, tales como la organización y trabajo en equipo, responsabilidad, creatividad, organización individual, adquirir conocimientos acerca de la migración e interculturalidad, cultura de diferentes países, búsqueda de información y trabajar con el uso de las TIC, rubro en el que destacaron las herramientas ofimáticas.

A continuación, en la tabla 2, se presenta un ejemplo de un instrumento empleado para la autoevaluación del empleo de las cualidades de la expresión oral durante el desarrollo de las exposiciones de los noticieros. 
Tabla 2. Instrumento de autoevaluación

\begin{tabular}{|c|c|c|c|c|c|c|}
\hline Exposición oral & 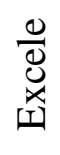 & $\stackrel{\gtrsim}{\stackrel{\Xi}{\Sigma}}$ & 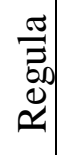 & 导 & z & 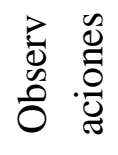 \\
\hline \multicolumn{7}{|l|}{ Cualidades de la expresión oral } \\
\hline \multicolumn{7}{|l|}{ Autodominio } \\
\hline \multicolumn{7}{|l|}{ Organización de las ideas } \\
\hline \multicolumn{7}{|l|}{ Fluidez } \\
\hline \multicolumn{7}{|l|}{ Coherencia } \\
\hline \multicolumn{7}{|l|}{ Sencillez } \\
\hline \multicolumn{7}{|l|}{ Claridad } \\
\hline \multicolumn{7}{|l|}{ Movimientos corporales } \\
\hline \multicolumn{7}{|l|}{ Tono y modulación de la voz } \\
\hline \multicolumn{7}{|l|}{ Comunicación no verbal } \\
\hline \multicolumn{7}{|l|}{ Posición del cuerpo } \\
\hline \multicolumn{7}{|l|}{ Expresión del rostro } \\
\hline \multicolumn{7}{|l|}{ Movimiento de las manos } \\
\hline \multicolumn{7}{|l|}{ Manejo del tema } \\
\hline \multicolumn{7}{|l|}{ Domina el tema } \\
\hline \multicolumn{7}{|l|}{ Organiza de ideas } \\
\hline \multicolumn{7}{|l|}{ Presenta de hechos para sustentar las ideas } \\
\hline \multicolumn{7}{|l|}{ Comunicación con el grupo } \\
\hline \multicolumn{7}{|l|}{ Realiza preguntas } \\
\hline \multicolumn{7}{|l|}{ Es mesurado en sus planteamientos } \\
\hline \multicolumn{7}{|l|}{ Resuelve correctamente las preguntas planteadas } \\
\hline \multicolumn{7}{|l|}{ Ejemplifica } \\
\hline TOTAL & & & & & & \\
\hline
\end{tabular}

Fuente: Elaboración propia

\section{Población y muestra}

La población del estudio estuvo integrada por los estudiantes de educación media superior de primer semestre en un centro educativo impartido por el IPN. Para hallar el tamaño óptimo de la muestra se empleó el procedimiento recomendado por Martínez (2012), con base en las siguientes condiciones: una población de 120 alumnos de primer semestre de bachillerato, una probabilidad de éxito de $0.5(p)$, un nivel de confianza de $95 \%$. Siguiendo estos parámetros se obtuvo una muestra representativa de 88 participantes. 


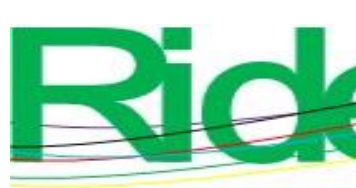

Revista Iberoamericana para la

Investigación y el Desarrollo Educativo

ISSN $2007-7467$

\section{Instrumento}

El instrumento que se diseñó para obtener la información fue un cuestionario basado en una escala Likert y organizado de la siguiente manera: 1) Datos sociodemográficos del participante y 2) Nivel de frecuencia en el desarrollo de las habilidades blandas organizadas en cuatro categorías: Interpersonales, Toma de decisiones, Pensamiento crítico y de autocontrol y Habilidades comunicativas. Los ítems se midieron a través de una escala de frecuencia de cinco grados que fueron: muy frecuentemente, frecuentemente, ocasionalmente, raramente y nunca. El instrumento fue sometido a un juicio de expertos a fin de determinar la validez de su contenido para después obtener su fiabilidad y consistencia interna mediante el coeficiente alfa de Cronbach, la cual fue de 0.811 , por lo que se consideró confiable. En tanto, la aplicación del instrumento se realizó de forma presencial, se brindó la información sobre la investigación a los participantes y se subrayó la confidencialidad de los datos proporcionados.

Una vez realizado el trabajo de campo, se procedió a organizar la información recabada del instrumento con la finalidad de llevar a cabo el análisis descriptivo para determinar el nivel de desarrollo de las habilidades blandas en el estudiantado de educación media superior.

\section{Resultados}

En lo que respecta al género de la muestra del estudio, se distribuyó del siguiente modo: 59 hombres y 29 mujeres, esto es, en términos porcentuales, $67 \%$ y $33 \%$, respectivamente.

El desarrollo de las habilidades blandas a partir del empleo de la metodología del AOP se describe a continuación.

Figura 2. Habilidades interpersonales y de comunicación

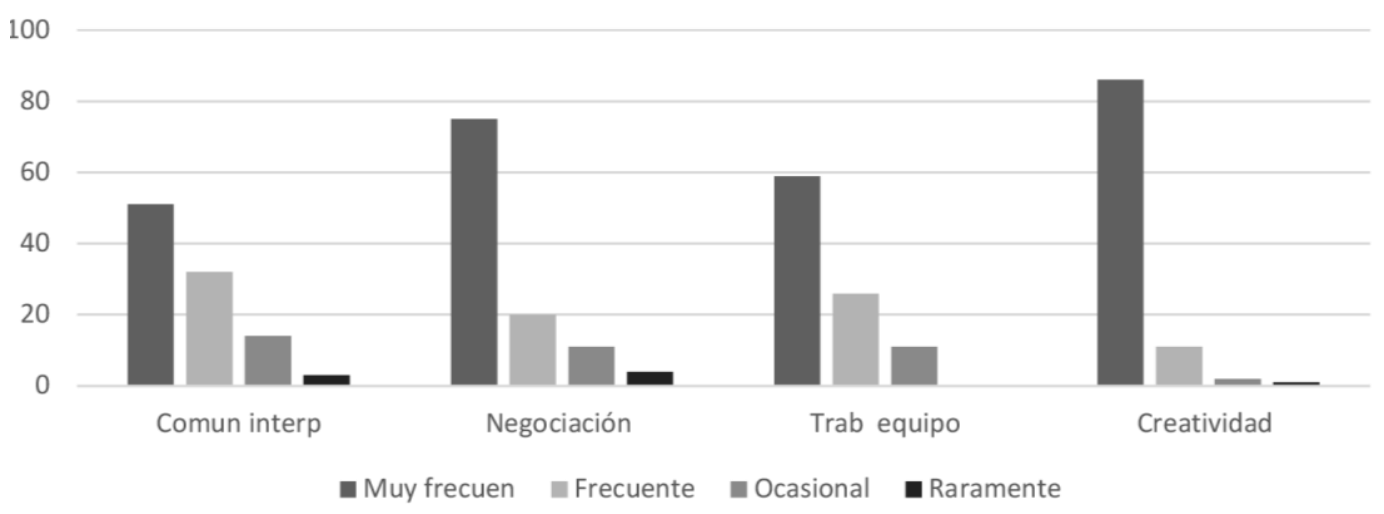

Fuente: Elaboración propia

Con base en la figura 2, se encontró que $56 \%$ del estudiantado muy frecuentemente desarrolló sus habilidades de comunicación interpersonal; 88 \% consolidó la negociación; 67 $\%$ reconoció la relevancia del trabajo en equipo, y $93 \%$ lo relacionado con la creatividad. Por tanto, la mayoría de los encuestados afirmó que la estrategia didáctica basada en el AOP 


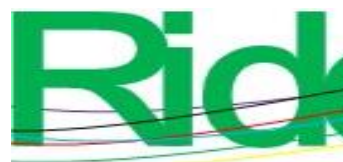

Revista Iberoamericana para la Investigación y el Desarrollo Educativo ISSN $2007-7467$

favoreció el desarrollo de las habilidades interpersonales y de comunicación en este nivel educativo.

Este hallazgo confirma lo encontrado por la investigación realizada por Palazuelos et al. (2018), Sánchez (2018), Landron et al. (2018) y Toledo y Sánchez (2018), quienes determinaron que el AOP es una metodología activa para la formación de conocimientos, habilidades, actitudes y valores en el estudiantado, y en consecuencia la catalogaron como una propuesta que tiene un impacto en el aprovechamiento académico.

Figura 3. Habilidades de toma de decisión y pensamiento crítico

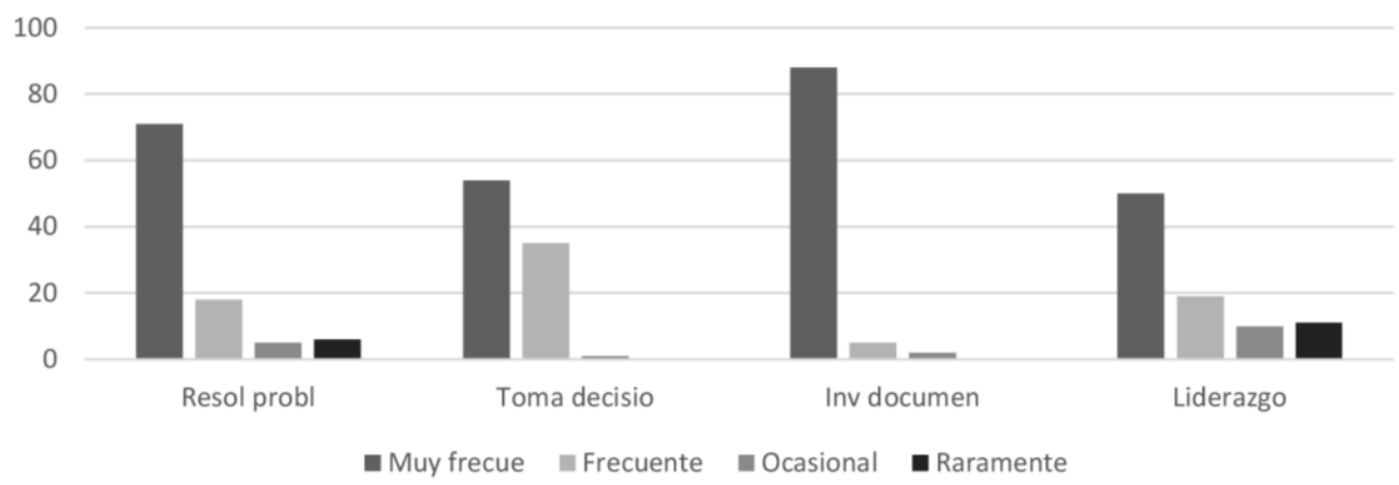

Fuente: Elaboración propia

Como se puede observar en la figura 3, el desarrollo de las habilidades relacionadas a la toma de decisiones y el pensamiento crítico, según los participantes, fue preponderantemente muy frecuente: la investigación documental en $88 \%$, la resolución de problemas en $71 \%$, toma de decisiones en $54 \%$ y el liderazgo en $50 \%$. Respecto a estas mismas habilidades, $19 \%$ manifestó haberlas desarrollado frecuentemente. Por consiguiente, se infiere que la metodología basada en el AOP favorece estas competencias, lo cual permitirá un incremento en las habilidades del pensamiento complejo de síntesis y análisis.

Estos resultados secundan lo hallado tanto por Rodríguez et al. (2018) como por Rico et al. (2018), quienes determinaron que el AOP tiene un efecto positivo en la formación del estudiantado en lo concerniente al desarrollo de las habilidades de investigación para la resolución de problemas.

También se corrobora lo encontrado por Alvarado et al. (2018), en cuyo estudio los participantes valoraron positivamente el uso del AOP para su formación académica debido a que les posibilitó la elaboración del proyecto y el desarrollo del pensamiento crítico. 


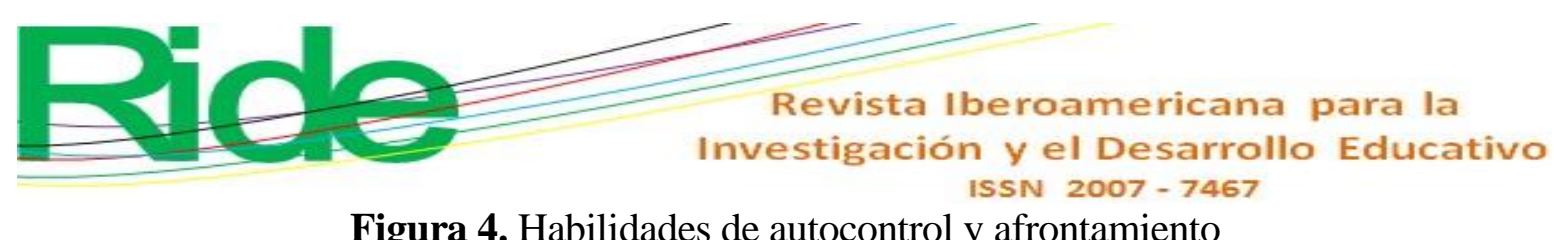

Figura 4. Habilidades de autocontrol y afrontamiento

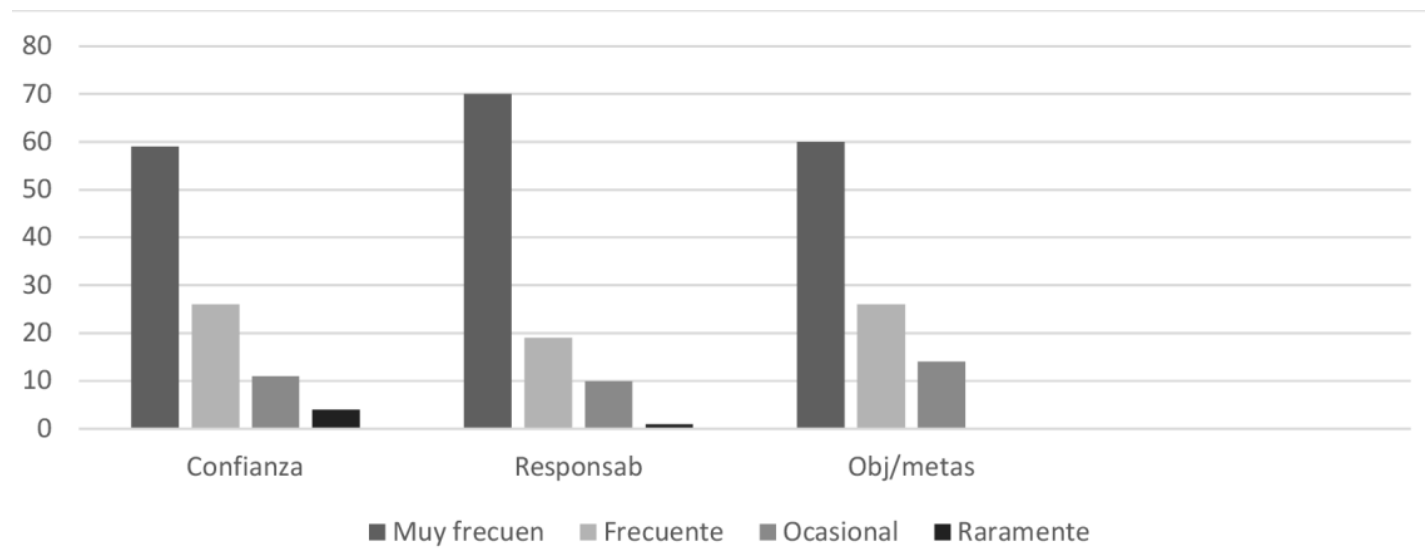

Fuente: Elaboración propia

La figura 4, por su parte, muestra gráficamente el resultado de las habilidades de autocontrol y afrontamiento. Al respecto, el alumnado indicó un desarrollo muy frecuente en la responsabilidad (70 \%), en fijarse objetivos y metas $(60 \%)$ y en aumentar la confianza personal (59\%). Por tanto, estas habilidades, al desarrollarse, permiten además lograr hábitos y valores que favorecen sus procesos individuales y de interacción social con los demás.

Estos datos respaldan lo establecido por Toledo y Sánchez (2018), González et al. (2018) y Landron et al. (2018) en lo referente a que el AOP desarrolla las competencias de la responsabilidad, interés y confianza, lo que conduce al logro del objetivo del proyecto a realizar.

Figura 5. Habilidades comunicativas desarrolladas

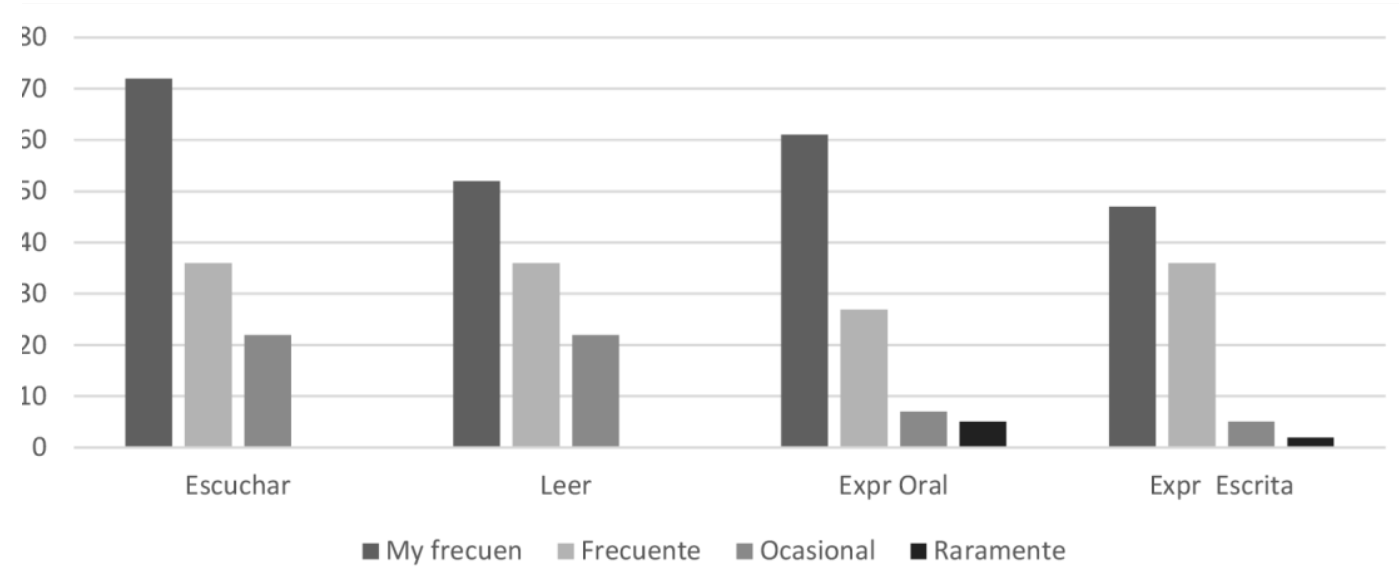

Fuente: Elaboración propia

Finalmente, en la figura 5 se encuentra graficado el resultado respecto al desarrollo de las habilidades comunicativas a través de la estrategia didáctica del AOP. Como puede ser apreciado, de manera muy frecuente los participantes destacaron el saber escuchar (72\%), la expresión oral (61 \%), la lectura (52\%) y la expresión escrita (47 \%). Así, este hallazgo demuestra la percepción de competencias genéricas o transversales necesarias en el desempeño de la vida diaria, académica y de tareas propias de cada profesión. 


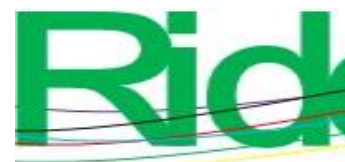

Revista lberoamericana para la

Investigación y el Desarrollo Educativo ISSN $2007-7467$

Dicho resultado corrobora lo hallado por Sánchez (2018), González et al. (2018) y Alvarado et al. (2018) en lo que se refiere a que el AOP favorece el desarrollo de la comunicación oral y escrita aunado a que contribuye a lograr un mejor análisis y argumentación del proyecto a realizar, debido a la lectura, intercambio de información y diseño de la estrategia de resolución del proyecto.

\section{Discusión}

Con base en los resultados obtenidos, la presente investigación confirma que el AOP fomenta el aprendizaje activo, en donde el alumno participa en la construcción de conocimientos disciplinares y el desarrollo de habilidades blandas, tal y como lo señalaron previamente Davcev et al. (2016).

En lo que respecta al desarrollo del trabajo colaborativo, integra actitudes que lo favorecen como la creatividad, la negociación con miras a tener experiencias similares a las laborales en un futuro, lo cual confirma lo encontrado tanto por Willard y Duffrin (2003) como por Carrasco et al. (2015).

De la misma manera, el uso del AOP, a lo largo de su implementación, integra ambientes de aprendizajes interdisciplinarios, mediados por las TIC, lo cual también implica identificar, analizar, discernir y seleccionar aquella información relevante para el proyecto, por lo que también se confirma lo hallado por Álvarez y Boillos (2015).

Por lo que, como lo establecen Konopka et al. (2015), es necesario incluir las TIC en la formación académica del alumnado para conseguir su mayor participación en el aprendizaje empleando una metodología didáctica activa como es el AOP.

No obstante, la metodología del AOP presenta limitaciones, fortalezas y debilidades, las cuales son importantes mencionarlas para dilucidar la mejor forma de enfrentarlas, así como permitir la realización de futuros estudios.

Las principales fortalezas son las siguientes:

- Las fortalezas encontradas confirman el carácter teórico-práctico para resolver el problema abordado.

- Se inicia con el conocimiento de un concepto relativamente nuevo: habilidades blandas.

- Los resultados de la investigación pueden motivar a integrar la metodología de AOP y a conceptualizar o reconceptualizar las funciones del docente y del alumno, así como del proceso enseñanza-aprendizaje.

En cuanto a las principales debilidades: 


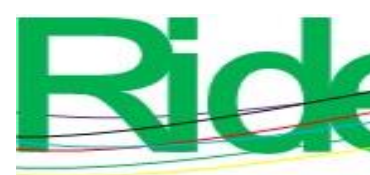

Revista Iberoamericana para la Investigación y el Desarrollo Educativo ISSN $2007-7467$

- $\quad$ El tiempo de seguimiento del proyecto fue de un semestre por los cambios de maestros a los grupos.

- La dificultad para socializar o mostrar los resultados a la comunidad.

Las principales limitaciones son las siguientes:

- $\quad$ El periodo para implementar la metodología de AOP fue de acuerdo con fechas oficiales de un semestre, en tiempo real de cuatro meses.

- Considerar el tamaño de la muestra, 88 alumnos fue el número mínimo de alumnos que integran la población, solo 2 grupos de 36.

- Dificultad de integrar programas vigentes desde 2008 con una metodología o estrategia de las más recientes e innovadoras, vencer las dificultades para la implantación de trabajo, evaluación y producto.

\section{Conclusiones}

Como se mencionó al inicio de este texto, el objetivo principal del estudio fue desarrollar en estudiantes de nivel medio superior del Instituto Politécnico Nacional habilidades blandas mediante la implementación de la metodología de AOP. Esto se logró debido a que reconocieron el logro de sus competencias en las cuatro categorías propuestas, a saber: Interpersonales, Toma de decisiones, Pensamiento crítico y de autocontrol y Habilidades comunicativas.

En este sentido, la relevancia del estudio fue proporcionar información trascendental para la educación media superior impartida por el IPN, según la percepción del alumnado, para así estar en posibilidad de implementar acciones formativas orientadas a la consolidación de las competencias blandas a partir del empleo de la metodología del AOP.

De este modo, la presente investigación confirma que el AOP fomenta el aprendizaje basado en el alumno, quien a su vez emprende la construcción del conocimiento aprendizaje sintiéndose motivado por ser él quien hace una propuesta o solución a una situación real, por ser quien descubre alguna información que no estaba a su alcance y, lo más importante, por descubrir habilidades y actitudes que no conocía.

También propicia el trabajo colaborativo integrando actitudes que lo favorecen, como la creatividad y la negociación, que le permita direccionar sus propias actividades, ejerciendo el liderazgo, aunado a un aprendizaje de y con otros.

De la misma manera, el AOP integra ambientes de aprendizaje interdisciplinarios y mediados por las TIC. Ambas herramientas incrementaron y nutrieron las tareas durante el proceso del proyecto, la información a investigar, y contribuyeron a la motivación y la comunicación de los participantes. 


\section{Referencias}

Al-Balushi, S. y Al-Amri, S. (2014). The effect of environmental science projects on students' environmental knowledge and science attitudes. International Research in Geographical and Environmental Educational, 23(3), 213-227.

Alonso, A. (2018). Aprendizaje basado en proyectos para el desarrollo de la competencia digital docente en la formación inicial del profesorado. Revista Latinoamericana de Tecnología Educativa, 17(1), 9-24.

Alvarado, H., Galindo, M. y Retamal, M. (2018). Evaluación del aprendizaje de la estadística orientada a proyectos en estudiantes de ingeniería. Educación Matemática, 30(3), 151183.

Álvarez, V., Herrejón, V., Morelos, M. y Rubio, M. (2010). Trabajo por proyectos: aprendizaje con sentido. Revista Iberoamericana de Educación, 52(5), 1-13.

Álvarez, M. y Boillos, M. (2015). La competencia comunicación escrita. En Villardón, L. (coord.), Competencias genéricas en educación superior. Metodologías específicas para su desarrollo. España: Narcea.

Austin, V., Abella, V., Delgado, V y Hortigüela, D. (2016). Aprendizaje Basado en Proyectos a través de las TIC. Una experiencia de innovación docente desde las aulas universitarias. Formación Universitaria, 9(3), 31-38.

Blanco, P., Jové, M. C. y Reverter, J. (2012). Paradigma estratégico para el desarrollo de habilidades competenciales Estudio descriptivo sobre la variabilidad en la percepción de habilidades competenciales de 40 alumnos de educación física en fase de formación inicial. Educación XX1, 15(2), 231-248.

Blanchard, M. y Dolores, M. (2016). Los proyectos de aprendizaje. Un marco metodológico para la innovación. Madrid, España: Narcea Ediciones.

Capilla, V R. (3 de octubre de 2017). ¿Qué es la industria 4.0? Agencia Informativa Conacyt. Recuperado de http://www.conacytprensa.mx/index.php/sociedad/politicacientifica/18282-la-industria-4-0.

Carrasco, A., Donoso, J. A., Duarte, T., Hernández, J. J. y López, R. (2015). Diseño y validación de un cuestionario que mide la percepción de efectividad del uso de metodologías de participación activa (CEMPA). El caso del Aprendizaje Basado en Proyectos (ABP) en la docencia de la contabilidad. Innovar, 25(58), 125-141.

Cascales, A. y Carrillo, M. (2018). Aprendizaje basado en proyectos en educación infantil: cambio pedagógico y social. Revista Iberoamericana de Educación, (76), 79-98. 


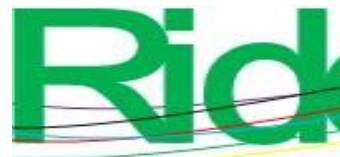

Coleman, G. (January 23, 2016). The next industry revolution will not be televised. World Economic Forum. Retrieved from www.weforum.org/agenda/2016/01/the-nextindustry-revolution-will-not-be-televised/.

Dahm, K., Farrell, S. and Ramachandran, R. (2015). Communication in the Engineering Curriculum: Learning to Write and Writing to Learn. Journal of Engineering Education Transformations, 29(2), 1-8.

Davcev, D., Stojkoska, B., Kalajdziski, S. and Trivodaliev, K. (2016). Project Based Learning of Embedded Systems. Retrieved from http://arxiv.org/abs/1606.07498.

David, H. (2015). Why are there still so many jobs? The history and future of workplace automation. The Journal of Economic Perspectives: A Journal of the American Economic Association, 29(3), 3-30.

De Miguel, M. (2006). Metodologías de enseñanza y aprendizaje para el desarrollo de competencias. Orientaciones para el profesorado universitario ante el Espacio Europeo de Educación Superior. Madrid, España: Alianza.

Dole, S., Bloom, L. and Kowalske, K. (2016). Transforming pedagogy: changing perspectives from teacher-centered to learner-centered. The Interdisciplinary Journal of ProblemBased Learning, 10(1), 1-11.

Doménech-Casal, J. (2018). Concepciones del alumnado de secundaria sobre energía. Una experiencia de Aprendizaje Basado en Proyectos con globos aerostáticos. Enseñanza de la Ciencias, 36(2), 191-213.

Escudero, N. (2018). Redefinición del "aprendizaje en red" en la cuarta revolución industrial. Apertura, 10(1), 149-163. Recuperado de http://dx.doi.org/10.18381/Ap.v10n1.1140.

Freeman, S., Eddy, S, McDonough, M., Smith, M., Okoroafor, N., Jordt, H. and Wenderoth, M. (2014). Active learning increases student performance in science engineering and mathematics. Proceedings of the National Academy of Sciences, 111(23), 8410-8415.

Frey, C. and Osborne, M. (2017). The future of employment: How susceptible are Jobs to computerisation? Technological Forecasting and Social Change, 114, 254-280.

González, V., Ferreira, J. y Barranco, A. (2018). Desarrollo de habilidades blandas y el uso del Sistema de Gestión del Aprendizaje en la elaboración de proyectos prácticos en una asignatura introductoria de Ingeniería Telemática. Cuaderno de Pedagogía Universitaria, 15(29), 44-53.

Hernando, M., Galán, R., Navarro, I. y Rodríguez, D. (2011). Ten Years of Cybertech: The Educational Benefits of Bullfighting Robotics. IEEE Transactions on Education, 54(4), 569-575. 


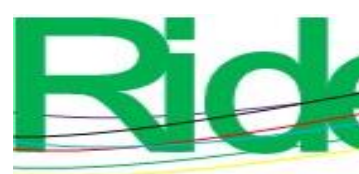

Revista Iberoamericana para la Investigación y el Desarrollo Educativo ISSN $2007-7467$

Joyanes, L. (2018). Industria 4.0. La cuarta revolución industrial. México: Alfaomega Editorial.

Konopka, C., Adaime, M. and Mosele, P. (2015). Active teaching and learning methodologies: some considerations. Creative Education, 6(14), 1536-1545.

Landron, M., Agreda, M. y Colmenero, M. (2018). El efecto del aprendizaje basado en proyectos en estudiantes con altas capacidades intelectuales de una segunda lengua. Revista de Educación, (380), 210-236.

Mafflioli, F. and Giuliano, A. (2003). Tuning Engineering education into the European higher education orchestra. European Journal of the Engineering Education, 28(3), 251-273. Retrieved from doi.org/10.1080/0304379031000098832.

Marcovitch, J. (2002). La universidad (im)posible. Cambridge, United Kingdom: Cambridge University Press.

Markham, T., Larmer, J. and Ravitz, J. (2003). Project-Based Learning Handbook: A Guide to Standards Focused Project-Based Learning for Middle and High School Teachers. Novato, United States: Buck Institute for Education.

Merrit, J., Lee, M., Rillero, P. and Kinach, B. (2017). Problem-Based Learning in K-8 Mathematics and Science Education: A Literature Review. Interdisciplinary Journal of Problem-Based Learning, 11(2), 1-13. Retrieved from https://doi.org/10.7771/15415015.1674 .

Mettas, A. and Constantinou, C. C. (2007). The technology fair: A project-based learning approach for enhancing problem solving skills and interest in design and technology education. International Journal of Technology and Design Education, 18(1), 79-100.

Murti, A. (2014). Why soft skills matter. IUP Journal of Soft Skills, 8(3), 32-36. Retrieved from https://papers.ssrn.com/sol3/papers.cfm?abstract_id=2636655.

Namakforoosh, J. M. (2011). Metodología de la investigación. México: Limusa.

Neebel, D., Merkel, C. and Wong, A. (2013). Engaging a variety of students in digital design with competition. In 2013 IEEE Frontiers in Education Conference (pp. 1091-1095).

Organización de las Naciones Unidas para la Educación, la Ciencia y la Cultura [Unesco]. (2015). Replantear la educación. ¿Hacia un bien común mundial? Organización de las Naciones Unidas para la Educación, la Ciencia y la Cultura. Recuperado de https://unesdoc.unesco.org/ark:/48223/pf0000232697.

Organisation for Economic Co-operation and Development [OECD]. (2012). Better Skills, Better Jobs, Better Lives: A Strategic Approach to Skills Policies. Paris, France: OECD Publishing. Retrieved from http://dx.doi.org/10.1787/9789264177338-en. 


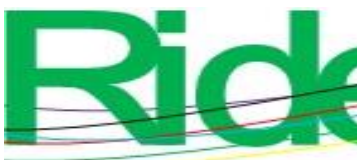

Revista Iberoamericana para la Investigación y el Desarrollo Educativo ISSN $2007-7467$

Organisation for Economic Co-operation and Development [OECD]. (2017). OECD Skills

Outlook 2017: Skills and Global Value Chains. Paris, France: OECD Publishing. Retrieved from https://dx.doi.org/10.1787/9789264273351-en.

Organización para la Cooperación y el Desarrollo Económicos [OECD, por sus siglas en inglés]. (2017). Diagnóstico de la OCDE sobre la Estrategia de Competencias, Destrezas y Habilidades de México. Resumen Ejecutivo. México: OECD. Recuperado de http://www.oecd.org/mexico/OECD-Skills-Strategy-Diagnostic-ReportMexico.pdf.

Ortega, S., Febles, R. y Estrada, S. (2016). Una estrategia para la formación de competencias blandas desde edades tempranas. Revista Cubana de Educación Superior, 35(2), 35-41. Recuperado de http://scielo.sld.cu/scielo.php?script=sci_arttext\&pid=S025743142016000200003\&lng=es\&tlng=es.(2016).

Palazuelos, E., San-Martín, P., Montoya, J. y Fernández, A. (2018). Utilidad percibida del Aprendizaje Orientado a Proyectos para la formación de competencias. Revista de Contabilidad, 21(2), 150-161.

Rico, B., Garay, L. y Ruiz, E. (2018). Implementación del aprendizaje basado en proyectos como herramienta en asignaturas de ingeniería aplicada. Revista Iberoamericana para la Investigación y el Desarrollo Educativo, 9(17), 20-57. https://doi.org/10.23913/ride.v9i17.372

Rodríguez, F., Vitvitskaya, O. y Silva, M. (2018). Aprendizaje basado en proyectos en el nivel de competencias investigativas en estudiantes de Instituto Pedagógico, Trujillo, 2017. In Crescendo, 9(2), 181-199.

Roig, R. y Álvarez, J. (2019). Repercusión en Twitter de las metodologías activas ABP, Flipped Classroom y Gamificación. RIED, Revista Iberoamericana de Educación a Distancia, 22(2), 79-96.

Rosa, D. y Martínez, M. (2016). El trabajo cooperativo con las TIC para el tratamiento de contenidos de Biología con alumnos de 14-15 años. Journal of Science Education, 17(2), 69-74.

Sánchez, N. (2018). Clase invertida y aprendizaje basado en proyectos en el aula de biología: Un proyecto de innovación para $1^{\circ}$ de ESO. Valoración de la experiencia. Enseñanza \& Teaching, 36(1), 81-110.

Secretaría de Estrategias Industriales de Madrid. (2017). La Digitalización y la Industria 4.0. Impacto industrial y laboral. Madrid, España: Secretaría de Estrategias Industriales de Madrid. Recuperado de http://www.industria.ccoo.es. 


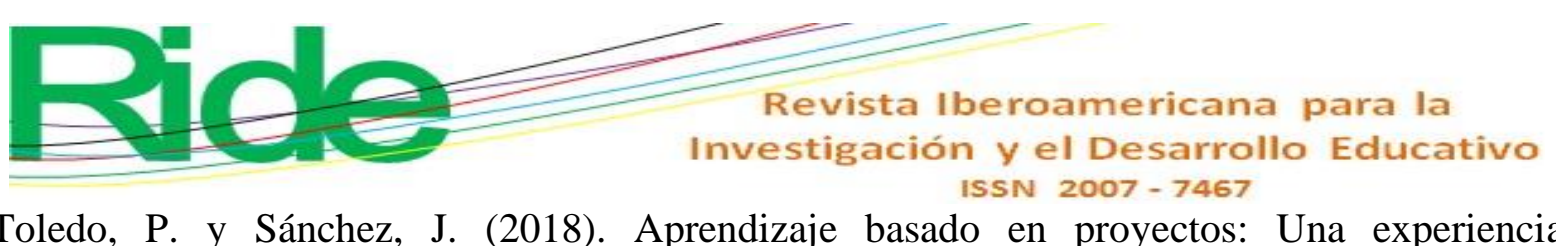

Toledo, P. y Sánchez, J. (2018). Aprendizaje basado en proyectos: Una experiencia universitaria. Profesorado, Revista de currículum y formación del profesorado, 22(2), 471-491.

Thomas, J. (2010). A review of research on Project-based learning. California, United States: The Autodesk Foundation.

Vergara, J. (2015). Aprendo porque quiero. El Aprendizaje Basado en Proyectos, paso a paso. México: Editorial SM.

Ynzunza, C., Izar, L., Bocarando, C., Aguilar, P. y Larios, O. (2017). El Entorno de la Industria 4.0: Implicaciones y Perspectivas Futuras. Conciencia Tecnológica, (54). Recuperado de https://www.redalyc.org/articulo.oa?id=94454631006.

Willard, K. and Duffrin, M. W. (2003). Utilizing project-based learning and competition to develop student skills and interest in producing quality food items. Journal of Food Science Education, 2(4), 69-73.

World Economic Forum [WEF]. (2016). The future of jobs. Employment, Skills and workforce strategy for the Fourth Industrial Revolution. World Economic Forum. Recuperado de http://www3.weforum.org/docs/WEF.

World Economic Forum [WEF]. (2017). Project Plan Overview: 21st Century Curriculum Project. Sydney, Australia: World Economic Forum. 


\begin{tabular}{|l|l|}
\hline Rol de Contribución & Autor (es) \\
\hline Conceptualización & María Elena (igual), Edgar Oliver (igual) y Jésica Alhelí (igual) \\
\hline Metodología & Edgar Oliver (igual) y Jésica Alhelí (igual) \\
\hline Software & Edgar Oliver \\
\hline Validación & Jésica Alhelí \\
\hline Análisis Formal & María Elena (igual) y Jésica Alhelí (igual) \\
\hline Investigación & María Elena (igual), Edgar Oliver (igual) y Jésica Alhelí (igual) \\
\hline Recursos & María Elena (igual), Edgar Oliver (igual) y Jésica Alhelí (igual) \\
\hline Curación de datos & Edgar Oliver \\
\hline $\begin{array}{l}\text { Escritura - Preparación del } \\
\text { borrador original }\end{array}$ & María Elena (igual), Edgar Oliver (igual) y Jésica Alhelí (igual) \\
\hline Escritura - Revisión y edición & María Elena (igual), Edgar Oliver (igual) y Jésica Alhelí (igual) \\
\hline Visualización & María Elena \\
\hline Supervisión & Edgar Oliver \\
\hline Administración de Proyectos & María Elena (igual), Edgar Oliver (igual) y Jésica Alhelí (igual) \\
\hline Adquisición de fondos & María Elena (igual), Edgar Oliver (igual) y Jésica Alhelí (igual) \\
\hline
\end{tabular}

